\title{
LBJMR medium: a new polyvalent culture medium for isolating and selecting vancomycin and colistin-resistant bacteria
}

\author{
Lucie Bardet ${ }^{\dagger}$, Stéphanie Le Page ${ }^{\dagger}$, Thongpan Leangapichart and Jean-Marc Rolain ${ }^{*}$ (D)
}

\begin{abstract}
Background: Multi-drug resistant bacteria are a phenomenon which is on the increase around the world, particularly with the emergence of colistin-resistant Enterobacteriaceae and vancomycin-resistant enterococci strains. The recent discovery of a plasmid-mediated colistin resistance with the description of the transferable mcr-1 gene raised concerns about the need for an efficient detection method for these pathogens, to isolate infected patients as early as possible. The LBJMR medium was developed to screen for all polymyxin-resistant Gram-negative bacteria, including mcr-1 positive isolates, and vancomycin-resistant Gram-positive bacteria.

Results: The LBJMR medium was developed by adding colistin sulfate salt at a low concentration $(4 \mu \mathrm{g} / \mathrm{mL})$ and vancomycin $(50 \mu \mathrm{g} / \mathrm{mL})$, with glucose $(7.5 \mathrm{~g} / \mathrm{L})$ as a fermentative substrate, to a Purple Agar Base $(31 \mathrm{~g} / \mathrm{L})$. A total of 143 bacterial strains were used to evaluate this universal culture medium, and the sensitivity and specificity of detection were $100 \%$ for the growth of resistant strains. 68 stool samples were cultured on LBJMR, and both colistin-resistant Gram-negative and vancomycin-resistant Gram-positive strains were specifically detected.
\end{abstract}

Conclusions: The LBJMR medium is a multipurpose selective medium which makes it possible to identify bacteria of interest from clinical samples and to isolate contaminated patients in hospital settings. This is a simple medium that could be easily used for screening in clinical microbiology laboratories.

Keywords: Colistin resistance, Culture media, Mcr-1, mcr genes, Vancomycin-resistant enterococci, Enterobacteriaceae, Multi-drug resistant, Detection method, Screening method

\section{Background}

The worldwide emergence of multidrug-resistant (MDR) bacteria represents a major public health issue. Controlling the spread of these bacteria relies upon both reducing the prescription of antibiotics and preventing transmission from carrier patients to others [1]. More specifically, this prevention targets the emerging carbapenemase-producing Enterobacteriaceae and vancomycin-resistant enterococci (VRE) strains, with the development of specific detection methods, mostly based on chromogenic and selective culture media [2].

The increase in infections due to carbapenemaseproducing Enterobacteriaceae has led to the revival of

\footnotetext{
* Correspondence: jean-marc.rolain@univ-amu.fr

${ }^{\dagger}$ Equal contributors

URMITE, Aix Marseille Université UM63, CNRS 7278, IRD 198, INSERM 1095 IHU - Méditerranée Infection, 19-21 Boulevard Jean Moulin, 13385 Marseille Cedex 05, France
}

colistin as a last-resort treatment [3]. Its widespread use, particularly in the livestock food in many countries [4], has inevitably led to the emergence of colistin-resistant strains over the past ten years $[5,6]$. Until recently, all colistin resistance mechanisms which had been described were attributed to chromosomic mutations [7]. In China in 2015, Liu and colleagues were the first to report the plasmid-mediated colistin-resistance gene in animals and humans, which they named $m c r-1$ [8]. This was followed by descriptions of the variants $m c r-1.2$ and $m c r-1.3[9,10]$, $m c r-2, m c r-3, m c r-4$ and $m c r-5$ genes [11-14]. A significant number of human cases (in the majority of cases asymptomatic carriers) was described $[15,16]$. Particularly, the worldwide analysis of colistin-resistant strains with an unknown mechanism allowed the detection of a consequent number of mor-1-positive bacteria [17-19]. This mobile colistin resistance gene presents low resistance levels, with minimal inhibitory concentrations (MIC) 
of colistin around $4 \mu \mathrm{g} / \mathrm{ml}$, which is close to the clinical breakpoint of colistin resistance ( $>2 \mu \mathrm{g} / \mathrm{mL}$ ), according to the European Committee on Antimicrobial Susceptibility Testing (EUCAST) [20]. Those data raised concerns about the detection and isolation of these pathogens, and the screening of the $\mathrm{mcr}-1$ gene into carbapenemase-producing bacteria has been added to recommendations for clinical microbiology laboratories in France [21]. While carbapenemase screening is well-defined with phenotypic and molecular techniques [22], there is a need for colistinresistance screening tools [2]. Given the diversity of mechanisms of colistin resistance [23], phenotypic methods, such as chromogenic culture media $[2,24]$ are preferred for a rapid detection.

In addition to colistin-resistant Enterobacteriaceae, the detection of vancomycin-resistant enterococci isolates is of clinical concern. The prevalence of VRE strains is increasing in Europe, especially Enterococcus faecium [25] and has led to nosocomial infections in the United States [26], and their dissemination is associated with high mortality rates [27]. The development of an effective screening tool for the early detection of those multidrug resistant pathogens has become a priority, in order to adapt treatment and isolate patients who are either infected or carriers.

For such purposes, we developed a polyvalent selective culture medium which can isolate both colistin-resistant Gram-negative bacteria, including Enterobacteriaceae strains harboring the $m c r-1$ gene, and vancomycin-resistant Gram-positive bacteria. In this study, we evaluated the performances of this medium on a representative set of bacterial strains and fecal samples. This universal culture medium was named LBJMR, standing for Lucie Bardet-Jean-Marc Rolain, and has recently been patented [28].

\section{Methods}

\section{Bacterial strains and samples cultured on LBJMR}

A total of 143 bacterial strains were used in this study, of which 101 were Enterobacteriaceae: 75 with an acquired mechanism of resistance to colistin (including 32 strains harboring the $m c r-1$ gene $[6,19,29-37]$ and 43 to another resistance mechanism), six with an intrinsic colistin resistance mechanism [38], and 20 colistin-susceptible strains (Additional file 1: Table S1). This set also comprised 17 non-fermentative Gram-negative bacteria: ten were colistin-resistant strains [39] including one Pseudomonas aeruginosa, one Acinetobacter baumannii [39] and other pathogens that are frequently diagnosed in samples from patients with cystic fibrosis, and seven colistin-susceptible strains [40, 41]. Finally, 25 Gram-positive strains, including eight vancomycin-resistant enterococci [42], three intrinsically vancomycin-resistant genera and 14 vancomycinsusceptible strains [43] were also included in this study (Additional file 1: Table S1).
The colistin-resistance genes which were potentially involved were previously screened by RT-PCR for Enterobacteriaceae $[6,44]$, as were the vancomycin resistance genes vanA and $v a n B$ for the VRE strains [45]. MICs of colistin and vancomycin were determined using E-test $^{\circ}$ strips (BioMérieux, Marcy l'Étoile, France) for Gram-negative and Gram-positive strains, respectively, and MICs of daptomycin were determined for the four reference strains of Enterococcus faecium (DSM 17050, 25,698, 25,697 and 13,590), and were evaluated according to the EUCAST guidelines [20].

In addition, 68 samples were cultivated on LBJMR medium, including 66 stool samples (56 from humans and ten from chickens) that were previously screened for $m c r-1$ by RT-PCR (56 positive and ten negative) and two clinical rectal swabs obtained from patients in the La Timone hospital, from which a vancomycin-resistant E. faecium VRE strain was isolated. Colonies with different morphologies isolated by culture of samples on LBJMR were isolated by subculture on Trypticase Soy Agar (TSA, Becton-Dickinson, Heidelberg, Germany), and the species were identified using Matrix-Assisted Laser DesorptionIonization-Time Of Flight Mass Spectrometry (MALDITOF MS), as described previously [46].

\section{LBJMR development}

In order to choose the most suitable medium for selecting colistin-resistant Enterobacteriaceae, including strains harboring the $m c r-1$ gene, five commercial differential media usually used for the detection of Enterobacteriaceae were supplemented with a range of concentration of colistin sulfate salt (MP Biomedicals, Illkirch, France) from 0 to $32 \mu \mathrm{g} / \mathrm{mL}$ : Drigalski Lactose Agar (Biokar diagnostics, France), BBL ${ }^{\mathrm{Tx}}$ Eosin Methylene Blue Agar (EMB; BectonDickinson, Heidelberg, Germany), Difco ${ }^{\text {mi }}$ Violet Red Bile Agar (Becton-Dickinson, Heidelberg, Germany), BBL ${ }^{\mathrm{mu}}$ MacConkey Agar II (Becton-Dickinson, Heidelberg, Germany), and Difco ${ }^{\mathrm{Tw}}$ Purple Agar Base (Becton-Dickinson, Heidelberg, Germany) supplemented with $7.5 \mathrm{~g} / \mathrm{L}$ of glucose (MP Biomedicals, Illkirch, France). Four Klebsiella pneumoniae, including two colistin-resistant, and four Escherichia coli, including two which were colistin-resistant with the $m c r-1$ gene, were inoculated at $10^{6} \mathrm{CFU}$ on each prepared agar [19].

The Purple Agar Base supplemented with glucose $(7.5 \mathrm{~g} / \mathrm{L})$, colistin sulfate $(0,4$ and $8 \mu \mathrm{g} / \mathrm{mL})$ and vancomycin $(0$ and $50 \mu \mathrm{g} / \mathrm{mL}$ ) (Sandoz, Levallois-Perret, France) was evaluated with 24 representative strains, including 20 Enterobacteriaceae (five intrinsic genera, eight with acquired resistance and seven colistin-susceptible strains as control) and four Gram-positive strains susceptible to vancomycin, which were inoculated at $10^{6} \mathrm{CFU}$. This experiment was also conducted by replacing glucose with lactose (Laboratoires Humeau, La Chapelle-sur- 
Erdre, France). The optimum concentration of vancomycin for the medium composition was then determined using 20 Gram-positive strains representative of vancomycin resistance (three intrinsically-resistant genera and 17 enterococci, including seven VRE) and ten chicken stool samples from Algeria, five of which were positive for $m c r-1$ by RT-PCR, and five of which were negative $[19,44]$.

\section{LBJMR evaluation}

The sensitivity and specificity of the LBJMR medium to detect all colistin-resistant Gram-negative bacteria and all vancomycin-resistant Gram-positive bacteria were evaluated with all the strains listed in Additional file 1: Table S1 and samples listed in Additional file 1: Table S2, which were incubated on LBJMR in an aerobic atmosphere at $37{ }^{\circ} \mathrm{C}$ for between 24 and $48 \mathrm{~h}$.

Inocula of the strains were prepared at $0.5 \mathrm{McFarland}$ (corresponding to $1.5 \times 10^{8} \mathrm{CFU} / \mathrm{mL}$ according to the EUCAST expert system) and serial 10 -fold dilutions were then performed in Phosphate Buffer Saline. $10 \mu \mathrm{L}$ of these suspensions were deposited on agars and simultaneously on TSA medium to determine the detection limits for each bacterium by assessing the viable concentration by colony count. Growth or inhibition of these microorganisms was observed after overnight incubation at $37^{\circ} \mathrm{C}$ in an aerobic atmosphere. Those experiments were performed in duplicate.

Finally, as the LBJMR medium was developed so as to avoid Proteus swarming, Proteus mirabilis and Proteus vulgaris strains were inoculated alone or mixed with $E$. coli P10 $(m c r-1)$ at the same concentration to evaluate the isolation ability of the LBJMR medium. Cultures were incubated at $37^{\circ} \mathrm{C}$ for $72 \mathrm{~h}$.

\section{Mcr-1 screening on stool samples}

A total of 1052 stool samples were used in this study, 899 from humans from different regions of the world: Marseille $(n=212)$ and Angers $(n=128)$ in France, Thailand $(n=212)$, Laos $(n=189)$, Nigeria $(n=143)$, and Senegal $(n=15) .153$ samples were from animals, including chickens from Algeria $(n=10)$, rodents from Cambodia $(n=21)$, and rodents $(n=60)$, pigs $(n=16)$ and goats $(n=46)$ from Laos.

The presence of the $m c r-1$ gene in those stools was first screened using RT-PCR as previously described [44]. Briefly, the DNA of the stools was extracted, after pretreatment by proteinase $\mathrm{K}$ and incubation at $95{ }^{\circ} \mathrm{C}$, using Biorobot EZ1 Advanced XL (Qiagen, Hilden, Germany). Positive DNA was confirmed using standard PCR. Samples with positive DNA were then cultured on LBJMR, after a prior enrichment step performed by inoculating approximately $1 \mathrm{~g}$ of the sample in tryptic soy broth (TSB) medium (BioMérieux, Marcy l'Étoile, France) and incubating for
$24 \mathrm{~h}$ at $37^{\circ} \mathrm{C} .100 \mu \mathrm{L}$ of these liquid media were then plated on agars that were also incubated at $37^{\circ} \mathrm{C}$ for $24 \mathrm{~h}$.

For each Gram-negative isolate identified by MALDITOF MS, colistin MIC was determined with a colistin Etest ${ }^{\circ}$ strip. For each colistin-resistant isolate, the presence of $m c r-1$ was investigated by RT-PCR as previously described [44]. Susceptibility testing to 26 antibiotics was performed on $m c r-1$-positive strains using the disk diffusion method, and interpreted according to the EUCAST guidelines [20].

\section{Comparison of the LBJMR medium with other polymyxin- containing media}

The sensitivity and specificity of the LBJMR medium was first compared to the $\mathrm{BD}^{\mathrm{m}}$ Cepacia Medium (Cepacia, Becton-Dickinson, Heidelberg, Germany) medium with all the colistin-resistant Enterobacteriaceae strains listed in Additional file 1: Table S1. The LBJMR medium was then compared to the SuperPolymyxin medium with 103 bacterial strains, including 61 of the Enterobacteriaceae, 17 non-fermentative Gram-negative, and 25 Gram-positive strains (Additional file 1: Table S1). Other polymyxin-containing media were also compared concomitantly to the LBJMR medium, using EMB Agar (Sigma-Aldrich, Illkirch, Germany) as a control, with some of these strains as described below. Inocula were prepared following the same protocol as for the LBJMR evaluation.

Columbia Colistin Nalidixic Acid Agar $+5 \%$ sheep blood (CNA) (BioMérieux, Marcy l'Étoile, France) and three mixed culture media: EMB with colistin sulfate $(4 \mu \mathrm{g} / \mathrm{mL})$ and vancomycin $(50 \mu \mathrm{g} / \mathrm{mL})$, LBJMR with Daptomycin $(10 \mu \mathrm{g} / \mathrm{ml}$, Novartis, Horsham, United Kingdom) in place of vancomycin and LBJMR with Amphotericin B $(5 \mu \mathrm{g} / \mathrm{ml}$, Bristol-Myers Squibb, Rueil-Malmaison, France) were concomitantly tested with 45 Enterobacteriaceae strains representative of colistin resistance $(30 \mathrm{mcr}-1$, ten susceptible and five intrinsically resistant genera). LBJMR with Amphotericin B was also evaluated with the eight VRE strains. Finally, the LBJMR medium was compared to the SuperPolymyxin medium with 12 samples, including the two clinical rectal swabs from the La Timone hospital and ten human stools from Thailand, five of which had been identified as positive by culture for the detection of an isolate harboring the $m c r-1$ gene, and five controls that were negative for $\mathrm{mcr}-1$ using RT-PCR.

\section{Statistical analysis}

The data were analyzed using a Chi-test to compare the Cepacia and LBJMR media and a Student-t test for a pairwise comparison of SuperPolymyxin and LBJMR selective media for the detection of non-fermentative Gram-negative colistin-resistant strains. Significance was assessed at $p<0.05$. 


\section{Results}

\section{LBJMR development}

Because colistin is a cationic molecule, we looked for a medium which was deprived of any ions in its original composition and which was also deprived of any electrolytes to avoid Proteus spp. swarming. The optimal conditions for LBJMR medium (growth of colistin- and vancomycin-resistant strains and inhibition of colistinor vancomycin susceptible strains) were obtained on Purple Agar Base with glucose (Additional file 1: Table $\mathrm{S} 3), 4 \mu \mathrm{g} / \mathrm{mL}$ of colistin sulfate and $50 \mu \mathrm{g} / \mathrm{ml}$ of vancomycin (Additional file 1: Table S4). The culture of ten chicken stools from Algeria enabled the detection of $E$. coli strains harboring the $m c r-1$ gene from three of the five samples which were positive for $m c r-1$ by RT-PCR, including strain 235 which had previously been isolated on Cepacia medium [19] and no Gram-negative strains were detected from the five negative samples (Additional file 1: Table S2). A correlation was observed between the Ct-values that reflect the DNA concentration and the results of culture (Additional file 1: Table S2).

The final preparation of the LBJMR medium was as follows: $31 \mathrm{~g} / \mathrm{L}$ of Purple Agar Base, $7.5 \mathrm{~g} / \mathrm{L}$ of glucose, $4 \mu \mathrm{g} / \mathrm{mL}$ of colistin sulfate and $50 \mu \mathrm{g} / \mathrm{mL}$ of vancomycin. $7.5 \mathrm{~g} / \mathrm{L}$ of lactose was used in place of glucose for the 24 strains initially tested and the same results were obtained. Glucose was selected because, combined with bromocresol purple, a $\mathrm{pH}$ indicator, it revealed the polyvalent capacity of the LBJMR medium with an easy and fast visualization of the species of interest in the clinical routine: both Enterobacteriaceae and enterococci gave yellow colonies, contrasting on the purple agar and exhibiting different sizes $(2-3 \mathrm{~mm}$ and $0.1-1 \mathrm{~mm}$, respectively).

\section{LBJMR evaluation}

All the polymyxin-resistant Enterobacteriaceae strains $(n=81)$ tested were able to grow on the LBJMR medium, with the lowest detection limit $\left(10^{1} \mathrm{CFU}\right)$, including those with the $m c r-1$ gene, as well as all the colistin-resistant non-fermentative Gram-negative $(n=$ $10)$ and vancomycin-resistant Gram-positive strains ( $n=$ 11), with detection limits dependent on their MIC, as shown in Table 1. Meanwhile, all the bacteria susceptible to colistin or vancomycin were inhibited (not detected at $10^{5} \mathrm{CFU}$ ), giving rise to $100 \%$ sensitivity and specificity for the growth of Gram-negative colistin-resistant and Gram-positive vancomycin-resistant strains (Table 1). The swarming of Proteus sp. strains was fully inhibited on LBJMR, even after $48 \mathrm{~h}$ of incubation.

Of the 1052 stools screened for $m c r-1$ by RT-PCR, 66 were cultured on LBJMR, including 56 that were positive after 32 cycles of RT-PCR, as well as ten negative stool samples as controls. 16 cultures were positive on LBJMR medium, enabling the detection of 17 colistin-resistant Enterobacteriaceae strains harboring the $m c r-1$ gene (colistin MICs ranging from 2 to $8 \mu \mathrm{g} / \mathrm{mL}$ ): 15 were identified as $E$. coli and two as $K$. pneumoniae by MALDI-TOF MS (Additional file 1: Table S2). Indeed, two bacterial species, E. coli and $K$. pneumoniae, were detected from the culture of the same sample (FHM128) (Additional file 1: Table S2). Five isolates were already known from a previous study on Cepacia medium [6, 19]. All the $E$. coli isolates from France were ExtendedSpectrum Beta-Lactamase-producing strains (data not shown). In conclusion, the LBJMR medium enabled the isolation of new colistin-resistant strains harboring the $m c r-1$ gene. The weak culture results compared to PCR could be explained by the length of storage of the tested samples (up to four years) at $-80{ }^{\circ} \mathrm{C}$, possibly inducing the death of bacteria while their DNA was still detectable [47]. However, it should be noted that, for some samples, we isolated another kind of strain, mostly Gram-positive bacteria which have an intrinsic resistance to vancomycin, largely Pediococcus pentosaceus and Weissela cibaria, as shown in Additional file 1: Table S2.

Finally, the culture of two clinical samples from the hospital enabled the isolation of two vancomycin-resistant E. faecium isolates (VRE1 and VRE2). In addition, two colistin-resistant Enterobacteriaceae isolates were also detected in the VRE2 sample, identified as K. pneumoniae LB3 $(\mathrm{MIC}=64 \mu \mathrm{g} / \mathrm{mL})$ and $E$. coli $\mathrm{LB} 4(\mathrm{MIC}=16 \mu \mathrm{g} / \mathrm{mL})$, both negative for the $m c r-1$ gene (Fig. 1).

\section{Comparison between LBJMR and other selective polymyxin-containing media}

The LBJMR medium was more sensitive than the Cepacia and CNA media for detecting the strains exhibiting the mcr-1 gene (Additional file 1: Table S5). Indeed, only four of those strains could grow on the CNA medium, because it also contains nalidixic acid and fewer than half of them grew on the Cepacia medium. Comparison of the sensitivities of the LBJMR (100\%) and Cepacia (47\%) media using a Chi-square statistical test gave a significant $p$-value $\left(<10^{-4}\right)$.

LBJMR and SuperPolymyxin media presented the same sensitivity for Enterobacteriaceae, with the growth of all colistin-resistant strains (detection limit of $10^{1} \mathrm{CFU}$ ), including the $30 \mathrm{mcr}-1$ strains tested, and the inhibition of all susceptible strains (Table 1). In addition, the culture of ten human stools gave the same results: five were positive, with the detection of an $E$. coli positive for $m c r-1$, and the five controls remained negative (Additional file 1: Table S2). In contrast, the LBJMR medium showed a significantly higher sensitivity than the SuperPolymyxin medium for the detection of colistin-resistant non-fermentative Gram-negative strains, with a significant $p$-value of $0.019(<0.01)$ with a pairwise comparison using a Student-t test (Table 1). More 
Table 1 Detection limits of targeted bacteria on LBJMR and SuperPolymyxin culture media

\begin{tabular}{|c|c|c|c|c|}
\hline \multirow{2}{*}{$\frac{\text { Bacterial strains }}{\text { Gram-negatives }}$} & & \multirow[b]{2}{*}{ CT MIC } & \multicolumn{2}{|c|}{ Detection limits on culture media (CFU): } \\
\hline & & & LBJMR & SuperPolymyxin \\
\hline \multirow[t]{41}{*}{ Colistin-resistant Enterobacteriaceae } & E. coli SE65 & 4 & $10^{1}$ & $10^{1}$ \\
\hline & E. coli 117R & 4 & $10^{1}$ & $10^{1}$ \\
\hline & E. coli $1 \mathrm{R}$ & 4 & $10^{1}$ & $10^{1}$ \\
\hline & E. coli 1R 2104 & 4 & $10^{1}$ & $10^{1}$ \\
\hline & E. coli 44A & 4 & $10^{1}$ & $10^{1}$ \\
\hline & E. coli $6 \mathrm{R}$ & 4 & $10^{1}$ & $10^{1}$ \\
\hline & E. coli 85R & 4 & $10^{1}$ & $10^{1}$ \\
\hline & E. coli 95R & 4 & $10^{1}$ & $10^{1}$ \\
\hline & E. coli 96R & 4 & $10^{1}$ & $10^{1}$ \\
\hline & E. coli $134 \mathrm{R}$ & 3 & $10^{1}$ & $10^{1}$ \\
\hline & E. coli 143R & 3 & $10^{1}$ & $10^{1}$ \\
\hline & E. coli LH121 & 16 & $10^{1}$ & $10^{1}$ \\
\hline & E. coli $\mathrm{LH} 140^{1}$ & 12 & $10^{1}$ & $10^{1}$ \\
\hline & E. coli LH257 & 12 & $10^{1}$ & $10^{1}$ \\
\hline & E. coli $\mathrm{LH} 57^{1}$ & 8 & $10^{1}$ & $10^{1}$ \\
\hline & E. coli LH1 & 6 & $10^{1}$ & $10^{1}$ \\
\hline & E. coli LH30 & 6 & $10^{1}$ & $10^{1}$ \\
\hline & E. coli TH214 & 6 & $10^{1}$ & $10^{1}$ \\
\hline & E. coli TH99 & 4 & $10^{1}$ & $10^{1}$ \\
\hline & E. coli 235 & 4 & $10^{1}$ & $10^{1}$ \\
\hline & E. coli P6 & 6 & $10^{1}$ & $10^{1}$ \\
\hline & E. coli $\mathrm{P} 10$ & 4 & $10^{1}$ & $10^{1}$ \\
\hline & E. coli $\mathrm{P} 17$ & 4 & $10^{1}$ & $10^{1}$ \\
\hline & E. coli FHA $102^{2}$ & 12 & $10^{1}$ & $10^{1}$ \\
\hline & E. coli FHM19 ${ }^{3}$ & 12 & $10^{1}$ & $10^{1}$ \\
\hline & E. coli FHA $113^{4}$ & 12 & $10^{1}$ & $10^{1}$ \\
\hline & E. coli $\mathrm{NH} 4^{5}$ & 12 & $10^{1}$ & $10^{1}$ \\
\hline & E. coli TH176 & 6 & $10^{1}$ & $10^{1}$ \\
\hline & K. pneumoniae FHA60 & 8 & $10^{1}$ & $10^{1}$ \\
\hline & K. pneumoniae FHM128 & 4 & $10^{1}$ & $10^{1}$ \\
\hline & K. pneumoniae 119R & 3 & $10^{1}$ & $10^{1}$ \\
\hline & K. pneumoniae $\mathrm{LH} 131^{6}$ & 32 & $10^{1}$ & $10^{1}$ \\
\hline & K. pneumoniae $\mathrm{LH} 61^{7}$ & 24 & $10^{1}$ & $10^{1}$ \\
\hline & K. pneumoniae LH17 & 12 & $10^{1}$ & $10^{1}$ \\
\hline & K. pneumoniae LH92 & 12 & $10^{1}$ & $10^{1}$ \\
\hline & K. pneumoniae $\mathrm{LB} 1^{6}$ & 32 & $10^{1}$ & $10^{1}$ \\
\hline & P. mirabilis $\mathrm{FH} 112$ & $>256$ & $10^{1}$ & $10^{1}$ \\
\hline & P. vulgaris PV148 & $>256$ & $10^{1}$ & $10^{1}$ \\
\hline & P. alcalifaciens TH44 & $>256$ & $10^{1}$ & $10^{1}$ \\
\hline & M. morganii FM102 & $>256$ & $10^{1}$ & $10^{1}$ \\
\hline & S. marcescens E13 & 128 & $10^{1}$ & $10^{1}$ \\
\hline \multirow[t]{2}{*}{ Others colistin-resistant } & B. cepacia FHM-BC1 & 64 & $10^{3}$ & $10^{5}$ \\
\hline & B. серасіа FHM-BC2 & $>256$ & $10^{1}$ & $10^{1}$ \\
\hline
\end{tabular}


Table 1 Detection limits of targeted bacteria on LBJMR and SuperPolymyxin culture media (Continued)

\begin{tabular}{|c|c|c|c|c|}
\hline \multicolumn{3}{|l|}{ Bacterial strains } & \multicolumn{2}{|c|}{ Detection limits on culture media (CFU): } \\
\hline & A. xylosoxidans FHM-AX & 3 & $10^{1}$ & $>10^{5}$ \\
\hline & S. maltophilia FHM-SM & 12 & $10^{3}$ & $>10^{5}$ \\
\hline & I. limosus FHM-IL & $>256$ & $10^{4}$ & $10^{4}$ \\
\hline & P. pulmonicola FHM-PP & $>256$ & $10^{1}$ & $10^{3}$ \\
\hline & S. putrefaciens FHM-SP & NC & $10^{3}$ & $>10^{5}$ \\
\hline & O. anthropi FHM-OA & NC & $10^{2}$ & $>10^{5}$ \\
\hline & P. aeruginosa FHM-PACOLR1 & $>256$ & $10^{1}$ & $10^{1}$ \\
\hline & A. baumannii ABlsac_ColiR & 8 & $10^{2}$ & $10^{1}$ \\
\hline \multirow[t]{27}{*}{ Colistin-susceptible } & E. asburiae $\mathrm{P} 113$ & 0,19 & $>10^{5}$ & $>10^{5}$ \\
\hline & E. cloacae $\mathrm{NH} 151$ & 0,50 & $>10^{5}$ & $>10^{5}$ \\
\hline & E. cloacae $\mathrm{NH} 74$ & 0,38 & $>10^{5}$ & $>10^{5}$ \\
\hline & K. pneumoniae CIP 82.91 & 0,125 & $>10^{5}$ & $>10^{5}$ \\
\hline & K. pneumoniae LB2 & 0,128 & $>10^{5}$ & $>10^{5}$ \\
\hline & K. pneumoniae $\mathrm{TH} 20 \mathrm{~S}$ & 0,125 & $>10^{5}$ & $>10^{5}$ \\
\hline & K. pneumoniae $\mathrm{TH} 28 \mathrm{~S}$ & 0,125 & $>10^{5}$ & $>10^{5}$ \\
\hline & Proteus vulgaris P100 & 0,94 & $>10^{5}$ & $>10^{5}$ \\
\hline & S. enterica $108 \mathrm{R}$ & 1 & $>10^{5}$ & $>10^{5}$ \\
\hline & S. enterica $122 \mathrm{R}$ & 0,5 & $>10^{5}$ & $>10^{5}$ \\
\hline & E. coli ATCC 25922 CIP 76.24 & 0,094 & $>10^{5}$ & $>10^{5}$ \\
\hline & E. coli 161 & 0,094 & $>10^{5}$ & $>10^{5}$ \\
\hline & E. coli 169 & 0,094 & $>10^{5}$ & $>10^{5}$ \\
\hline & E. coli FHM88S & 0,125 & $>10^{5}$ & $>10^{5}$ \\
\hline & E. coli TH134S & 0,094 & $>10^{5}$ & $>10^{5}$ \\
\hline & E. coli LH53S & 0,094 & $>10^{5}$ & $>10^{5}$ \\
\hline & E. coli LH165S & 0,074 & $>10^{5}$ & $>10^{5}$ \\
\hline & E. coli TH77S & 0,064 & $>10^{5}$ & $>10^{5}$ \\
\hline & E. coli $282 \mathrm{~S}$ & 0,074 & $>10^{5}$ & $>10^{5}$ \\
\hline & E. coli FHM19S & 0,125 & $>10^{5}$ & $>10^{5}$ \\
\hline & P. aeruginosa FHM-PA4 & 2 & $>10^{5}$ & $>10^{5}$ \\
\hline & P. aeruginosa FHM-PA5 & 0,50 & $>10^{5}$ & $>10^{5}$ \\
\hline & P. aeruginosa FHM-PA6 & 0,38 & $>10^{5}$ & $>10^{5}$ \\
\hline & S. xianemensis $111 \mathrm{P}$ & 0,125 & $>10^{5}$ & $>10^{5}$ \\
\hline & S. xianemensis $111 \mathrm{~A}$ & 0,094 & $>10^{5}$ & $>10^{5}$ \\
\hline & A. nosocomialis ABG13S & 0,064 & $>10^{5}$ & $>10^{5}$ \\
\hline & A. pitti MK & 0,125 & $>10^{5}$ & $>10^{5}$ \\
\hline Gram-positives & & VCN MIC & & \\
\hline \multirow[t]{8}{*}{ Vancomycin-resistant } & E. faecium DSM17050 & $>256$ & $10^{1}$ & $>10^{5}$ \\
\hline & E. faecium DSM13590 & $>256$ & $10^{1}$ & $>10^{5}$ \\
\hline & E. faecium DSM25698 ${ }^{a}$ & $>256$ & $10^{2}$ & $>10^{5}$ \\
\hline & E. faecium DSM25697 & $>256$ & $10^{1}$ & $>10^{5}$ \\
\hline & E. faecium FHMVRE1 ${ }^{\mathrm{a}}$ & $>256$ & $10^{1}$ & $>10^{5}$ \\
\hline & E. faecium FHMVRE $2^{a}$ & $>256$ & $10^{1}$ & $>10^{5}$ \\
\hline & E. faecium VRE & 24 & $10^{3}$ & $>10^{5}$ \\
\hline & E. faecalis JH2-2: $\operatorname{Tn} 1549^{\mathrm{b}}$ & 64 & $10^{2}$ & $>10^{5}$ \\
\hline
\end{tabular}


Table 1 Detection limits of targeted bacteria on LBJMR and SuperPolymyxin culture media (Continued)

\begin{tabular}{|c|c|c|c|c|}
\hline \multirow[t]{2}{*}{ Bacterial strains } & \multirow[b]{2}{*}{ W. cibaria P18A } & \multirow[b]{2}{*}{$>256$} & \multicolumn{2}{|c|}{ Detection limits on culture media (CFU) } \\
\hline & & & $10^{1}$ & $>10^{5}$ \\
\hline & W. cibaria P18B & $>256$ & $10^{1}$ & $>10^{5}$ \\
\hline & L. lactis P18C & $>256$ & $10^{1}$ & $>10^{5}$ \\
\hline \multirow[t]{14}{*}{ Vancomycin-susceptible } & S. aureus CF_Marseille & 1 & $>10^{5}$ & $>10^{5}$ \\
\hline & E. faecium $\mathrm{TH} 26$ & 0,75 & $>10^{5}$ & $>10^{5}$ \\
\hline & E. faecium 349 & 0,75 & $>10^{5}$ & $>10^{5}$ \\
\hline & E. faecium $\mathrm{TH} 12$ & 2 & $>10^{5}$ & $>10^{5}$ \\
\hline & E. faecium LH165 & 0,75 & $>10^{5}$ & $>10^{5}$ \\
\hline & E. faecium Al7 & 0,75 & $>10^{5}$ & $>10^{5}$ \\
\hline & E. faecium $\mathrm{TH} 43$ & 0,75 & $>10^{5}$ & $>10^{5}$ \\
\hline & E. faecium TH95 & 0,75 & $>10^{5}$ & $>10^{5}$ \\
\hline & E. faecium 282 & 0,75 & $>10^{5}$ & $>10^{5}$ \\
\hline & E. faecalis $\mathrm{JH} 2-2: \mathrm{C} 2$ & 1,5 & $>10^{5}$ & $>10^{5}$ \\
\hline & E. faecalis $\mathrm{JH} 2-2 \mathrm{~S}$ & 1,5 & $>10^{5}$ & $>10^{5}$ \\
\hline & E. gallinarum SE3 & 1,5 & $>10^{5}$ & $>10^{5}$ \\
\hline & E. casseliflavus SE3 & 1,5 & $>10^{5}$ & $>10^{5}$ \\
\hline & E. hirae LH111 & 1 & $>10^{5}$ & $>10^{5}$ \\
\hline
\end{tabular}

MIC of colistin (CT) and vancomycin (VCN) are given in $\mu \mathrm{g} / \mathrm{mL}$. Enterobacteriaceae with $m c r-1$ gene are in bold font

Colistin-resistant strains with a mutation on another gene are indicated as follow: ${ }^{1} p h o P$ E375K, ${ }^{2} p m r B$ A159V, ${ }^{3} p m r B$ P7-Q12(del 6aa), ${ }^{4} p m r B$ T156 K,

${ }^{5} \mathrm{pmrB} 192$ insertion, ${ }^{6} \mathrm{mgrB}$ Stop, ${ }^{7} \mathrm{mgrB}$ Sub, ${ }^{8} \mathrm{pmrB}$ T157P. Enterococci strains with vancomycin-resistant genes are indicated as follows: ${ }^{\mathrm{a}} \mathrm{van} A$ and ${ }^{\mathrm{b}} \mathrm{van} B$

specifically, the LBJMR medium was able to detect those screened in the cystic fibrosis samples, such as Burkholderia cepacia, even at low concentrations, and could replace specific media such as the Cepacia medium for their isolation. The addition of amphotericin $\mathrm{B}$ or the replacement of vancomycin by daptomycin in the LBJMR medium did not affect the growth of Enterobacteriaceae strains (Additional file 1: Table S5). Finally, all the VRE strains were inhibited on the SuperPolymyxin medium because of the presence of daptomycin, while they were detected on the LBJMR medium (Table 1).
These results were confirmed by the culture of the two clinical samples from the hospital: vancomycin-resistant $E$. faecium could only grow on the LBJMR medium and not on the SuperPolymyxin medium for both samples, and the two Enterobacteriaceae from the VRE2 sample found on the LBJMR medium were also detected on the SuperPolymyxin medium.

Finally, the concomitant addition of colistin and vancomycin to EMB agar showed the systematic inhibition of $E$. coli strains harboring the $m c r-1$ gene, corresponding to the tested strains with the lowest MIC.

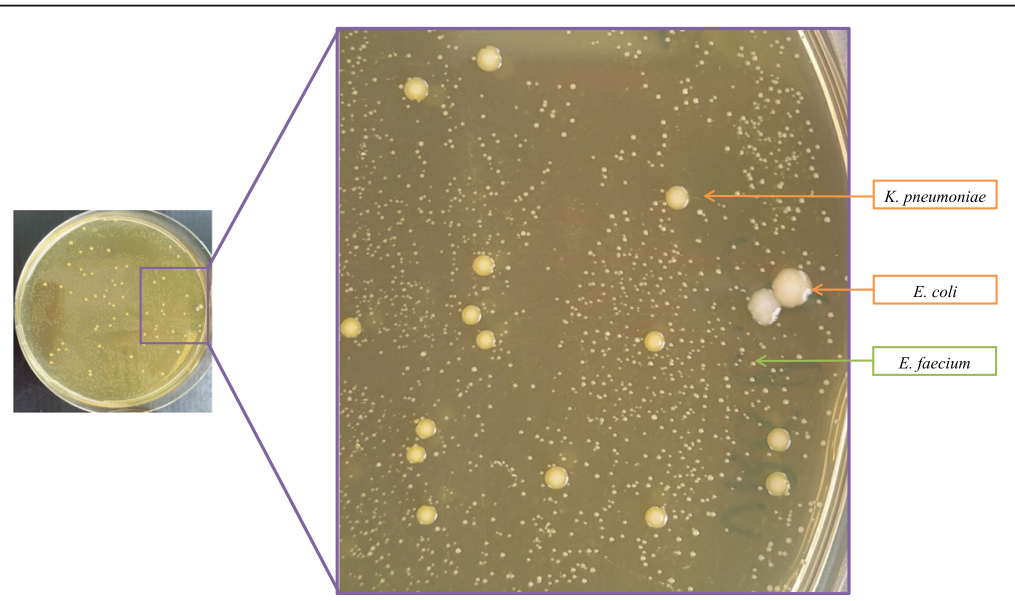

Fig. 1 Aspect of the different types of colonies on LBJMR after culture of a clinical sample. Enterobacteriaceae exhibited yellow colonies that are larger $(2-3 \mathrm{~mm})$ than enterococci $(0.1-1 \mathrm{~mm})$ 


\section{Discussion}

In accordance with recommendations aiming to isolate patients carrying multi-drug resistant bacteria, it is becoming essential to detect colistin-resistant Gramnegative pathogens, particularly with the recent description of plasmid-mediated colistin-resistant genes among Enterobacteriaceae strains [8-12]. We do not currently know the potential consequences of patient colonization by those resistant bacteria, as it has already been demonstrated that acquisition of resistance is associated with a decrease in virulence [48-50], but it is necessary to implement efficient tools to prevent and monitor as closely as possible any epidemic outbreak and develop a rapid therapeutic strategy.

Currently, the only available method for detecting colistin resistance, according to joint EUCAST and Clinical Laboratory Standard Institute (CLSI) expert systems, is antibiotic susceptibility testing using broth microdilution, which is not suitable for daily screening in clinical microbiology laboratories. Current polymyxin-containing culture media are also not appropriate because of their high concentration of polymyxin. Indeed, they were not developed to isolate Enterobacteriaceae but to specifically detect bacteria that are intrinsically resistant to polymyxins, avoiding contaminants such as Enterobacteriaceae species. Thus, the establishment of an effective protocol to detect colistin resistance with the development of a reliable culture medium was necessary in clinical microbiology laboratory.

The significant number of strains tested on our selective medium showed that the LBJMR medium allows all colistin-resistant Enterobacteriaceae strains to grow, even those with a very low MIC for colistin and harboring the mor-1 gene, when all the susceptible strains were inhibited. Furthermore, the screening conducted on stool samples that were positive for $m c r-1$ by PCR allowed the isolation of new $m c r-1$ strains (Additional file 1: Table S2). The LBJMR medium could also detect colistin-resistant non-fermentative Gram-negative bacteria, including pathogens which are often found in patients suffering from cystic fibrosis (Table 1). We thereby created a universal culture medium, able to replace specific polymyxincontaining culture media usually used in routine diagnosis. All the vancomycin-resistant bacteria tested were detected at low concentrations on the LBJMR medium, including the VRE strains, when susceptible were inhibited. To attest this capacity, the LBJMR medium should be tested on more VRE strains and comparatively to VRE selective culture media.

The performances of the LBJMR medium are summarized in Table 2: samples can be cultured directly on the LBJMR selective medium, without a previous decontamination step, and colonies can be directly analyzed from the primary culture, without subcultures. Bacteria of
Table 2 Performance of LBJMR medium

\begin{tabular}{ll}
\hline Criteria & LBJMR medium \\
\hline Isolates screened & Colistin-resistant Gram-negatives: \\
& - Enterobacteriaceae, including those harboring \\
& the mcr-1 gene \\
& - Non-fermentative Gram-negative colistin-resistant \\
& strains, including those involves in cystic fibrosis \\
& samples \\
& Vancomycin-resistant Gram positives, including \\
& Enterococci \\
& Yellow on purple agar: $2-3$ mm for \\
& Enterobacteriaceae, $0.1-1$ mm for Enterococci \\
Aspect of colonies & Aerobic atmosphere, $37{ }^{\circ} \mathrm{C}, 24 \mathrm{H}$ (sterile at 48H) \\
Incubation & Direct on LBJMR, no previous decontamination \\
Culture of samples & Colonies can be picked directly from primary \\
Isolates analysis & cultures on LBJMR for analysis: \\
& - MALDI-TOF identification \\
& - Antibiotic Susceptibility testing \\
& - PCR screening for resistance genes. \\
& - Inhibition of Proteus swarming \\
Avoid & - Inhibition of yeast possible by adding \\
contamination & amphotericin B \\
&
\end{tabular}

interest are easily recognizable, as shown in Fig. 1: both colistin-resistant Enterobacteriaceae and vancomycinresistant enterococci exhibit yellow colonies $(2-3 \mathrm{~mm}$ and $0.1-1 \mathrm{~mm}$ respectively), on a purple agar base. Because LBJMR also permits the growth of intrinsic resistant bacteria, screening should be completed with a rapid identification using MALDI-TOF MS [51], directly from the LBJMR medium. Antibiotic susceptibility testing and PCR screening can be performed in the same time.

Nordmann et al. developed a different selective medium called SuperPolymyxin, based on EMB agar and containing $3.5 \mu \mathrm{g} / \mathrm{mL}$ of colistin sulfate, $10 \mu \mathrm{g} / \mathrm{mL}$ of daptomycin and $5 \mu \mathrm{g} / \mathrm{mL}$ of amphotericin B [52], associated with a complementary phenotypic test, the rapid polymyxin NP [53], to screen for colistin-resistant Gram-negative bacteria [54]. These two media are differential and are able to recognize bacterial species based on color. Sensitivities for colistin-resistant Enterobacteriaceae were the same for the SuperPolymyxin and LBJMR media, but only the LBJMR medium could detect VRE strains (Table 1). Indeed, the SuperPolymyxin medium is composed of daptomycin and amphotericin B, which prevents the growth of contaminants including bacteria of interest such as VRE strains, because they are mostly susceptible to daptomycin. In our study, we tested stool samples and obtained some contaminants, including Gram-positive bacteria which were intrinsically resistant to vancomycin (Additional file 1: Table S2) or some yeasts.

More recently, the culture medium ChromAgar COLAPSE was developed and compared to the SuperPolymyxin medium [55]. The comparison of the LBJMR and the CHROMAgar COL-APSE performances have to be assessed. All those media should be also evaluated on 
heteroresistant strains, as they are difficult to detect and their frequence is widely underestimated [56].

The advantage of the LBJMR medium in terms of isolating vancomycin resistant enterococci can be also a disadvantage in a few cases, because Gram-positive bacteria which are intrinsically resistant to vancomycin bacteria, including Pediococcus, can also be isolated. In the LBJMR medium, Gram-positive bacteria appear with small colonies and a simple identification by MALDI-TOF can identify them. For the elimination of yeast in samples, amphotericin B can be added in our medium.

\section{Conclusion}

The LBJMR medium is an adequate screening tool for all colistin-resistant isolates from clinical samples, independently of their resistance level or mechanism. LBJMR could be used in routine laboratory work to detect colistinresistant and vancomycin-resistant bacteria, allowing for the direct analysis of colonies and, thus, the early isolation of contaminated patients in hospital settings. This medium is currently being investigated as a routine medium in our institute to determine its usefulness in detecting such bacteria.

\section{Additional file}

Additional file 1: Table S1. List of the studied strains. The MIC of colistin (CT) or vancomycin (VCN) are indicated in $\mu \mathrm{g} / \mathrm{mL}$. Table S2. List of stools used and Gram-negative isolates detected on LBJMR. Positive isolates for $\mathrm{mcr}-1$ and their corresponding samples are in bold font. New isolates harboring $\mathrm{mcr}-1$ are underlined. Their MICs of colistin are indicated in $\mu \mathrm{g} /$ $\mathrm{mL}$. (-) Negative (ND) fers to Not Done. Table S3. Comparison of different agar bases supplemented with different concentrations of colistin. Genes involved in colistin resistance are indicated in bold and colistin MICs are indicated in $\mu \mathrm{g} / \mathrm{mL}$. Table S4. Development of the selective medium for the detection of colistin-resistant strains. Table S5. Comparison of different polymyxin-containing media. Numbers indicate the total number of strains that grew on each culture media. (DOCX $99 \mathrm{~kb}$ )

\section{Abbreviations}

CLSI: Clinical Laboratory Standard Institute; CNA: Colistin Nalidixic Acid; EMB: Eosin-Methylene Blue; EUCAST: European Committee on Antimicrobial Susceptibility Testing; MALDI-TOF MS: Matrix-Assisted Laser Desorption-lonizationTime Of Flight Mass Spectrometry; MIC: Minimum Inhibitory Concentration; TSA: Trypticase Soya Agar; VRE: Vancomycin-Resistant Enterococci

\section{Acknowledgements}

We thank to Marielle Bedotto-Buffet for technical assistance and TradOnline for English corrections.

\section{Funding}

IHU Méditerranée Infection.

\section{Availability of data and materials}

Ok

\section{Authors' contributions}

LB designed the study, performed the experiments and analyzed and interpreted the data. SLP was analyzed, interpreted the data and involved in revising the manuscript. TL contributed to the development and analysis of data. JMR was responsible for the conception and design of the study, and the analysis of data. All authors read and approved the final manuscript.
Ethics approval and consent to participate

Not applicable

\section{Consent for publication}

Not applicable

\section{Competing interests}

The authors declare that they have no competing interests

\section{Publisher's Note}

Springer Nature remains neutral with regard to jurisdictional claims in published maps and institutional affiliations.

Received: 25 August 2017 Accepted: 15 November 2017

Published online: 23 November 2017

\section{References}

1. Haut Conseil de la Santé Publique. Prévention de la transmission croisée des Bactéries Hautement Résistantes aux antibiotiques émergentes ( BHRe ). 2013

2. Perry JDA. Decade of development of chromogenic culture media for clinical microbiology in an era of molecular diagnostics. Clin Microbiol Rev. 2017;30:449-79.

3. Biswas S, Brunel J-M, Dubus J-C, Reynaud-Gaubert M, Rolain J-M. Colistin: an update on the antibiotic of the 21st century. Expert Rev Anti-Infect Ther 2012:10:917-34

4. Olaitan AO, Morand S. Rolain J-MM. Emergence of colistin-resistant bacteria in humans without colistin usage: a new worry and cause for vigilance. Int J Antimicrob Agents. 2016;47:1-3.

5. Kempf I, Fleury MA, Drider D, et al. What do we know about resistance to colistin in Enterobacteriaceae in avian and pig production in Europe? Int J Antimicrob Agents. 2013;42:379-83

6. Olaitan AO, Diene SM, Kempf M, et al. Worldwide emergence of colistin resistance in Klebsiella pneumoniae from healthy humans and patients in Lao PDR, Thailand, Israel, Nigeria and France owing to inactivation of the PhoP/PhoQ regulator mgrB: an epidemiological and molecular stu. Int J Antimicrob Agents. 2014:44:500-7.

7. Olaitan AO, Morand S, Rolain J-M. Mechanisms of polymyxin resistance: acquired and intrinsic resistance in bacteria. Front Microbiol. 2014:5:643.

8. Liu Y-Y, Wang Y, Walsh TR, et al. Emergence of plasmid-mediated colistin resistance mechanism MCR-1 in animals and human beings in China: a microbiological and molecular biological study. Lancet Infect Dis. 2016;16: 161-8.

9. Di Pilato $V$, Arena F, Tascini $C$, et al. $m c r-1.2$, a new $m c r$ variant carried on a transferable plasmid from a colistin-resistant KPC carbapenemase-producing Klebsiella pneumoniae strain of sequence type 512. Antimicrob Agents Chemother. 2016;60:5612-5.

10. Yang $Y$-Q, Li Y-X, Song $T$, et al. Colistin resistance gene $m c r-1$ and its variant in Escherichia coli isolates from chickens in China. Antimicrob Agents Chemother. 2017;AAC:01204-16.

11. Xavier BB, Lammens $C$, Ruhal $R$, et al. Identification of a novel plasmidmediated colistin-resistance gene, $m c r-2$, in Escherichia coli, Belgium, June 2016. Euro Surveill. 2016;21:6-11.

12. Yin W, Li H, Shen Y, et al. Novel plasmid-mediated Colistin resistance gene mcr-3 in Escherichia coli. MBio. 2017:8:4-9.

13. Carattoli A, Villa L, Feudi $C$, et al. Novel plasmid-mediated colistin resistance mcr-4 gene in Salmonella and Escherichia coli, Italy 2013, Spain and Belgium, 2015 to 2016. Eur Secur. 2017;22:30589.

14. Borowiak M, Fischer J, Hammerl JA, Hendriksen RS, Szabo I, Malorny B. Identification of a novel transposon-associated phosphoethanolamine transferase gene, mcr-5, conferring colistin resistance in d-tartrate fermenting Salmonella enterica subsp. enterica serovar Paratyphi B. J Antimicrob Chemother 2017; published online Sept 18. DOl:10.1093/jac/dkx327.

15. Baron S, Hadjadj L, Rolain J-M, Olaitan AO. Molecular mechanisms of polymyxin resistance: knowns and unknowns. Int J Antimicrob Agents. 2016:48:583-91.

16. Poirel L, Jayol A, Nordmann P. Polymyxins: antibacterial activity, susceptibility testing, and resistance mechanisms encoded by plasmids or chromosomes. Clin Microbiol Rev. 2017;30:557-96.

17. Rolain J-M, Olaitan AO. Plasmid-mediated colistin resistance: the final blow to colistin? Int J Antimicrob Agents. 2016;47:4-5. 
18. Skov RL, Monnet DL. Plasmid-mediated colistin resistance ( $m c r-1$ gene): three months later, the story unfolds. Euro Surveill. 2016;21:30155.

19. Olaitan AO, Chabou S, Okdah L, Morand S, Rolain J-M. Dissemination of the mcr-1 colistin resistance gene. Lancet Infect Dis. 2016;16:147.

20. European Committee on Antimicrobial Susceptibility Testing (EUCAST). Breakpoint tables for interpretation of MICs and zone diameters. Version 71 2017; Version 7:: 0-94.

21. Haut Conseil de la santé publique. Avis relatif aux mesures à prendre en lien avec l'émergence d'une résistance plasmidique à la colistin $(m c r-1)$ chez les entérobactéries. 2016.

22. Bialvaei AZ, Kafil HS, Asgharzadeh M, Yousef Memar M, Yousefi M. Current methods for the identification of carbapenemases. J Chemother. 2016;28:1-19.

23. Jeannot K, Bolard A, Plésiat P. Resistance to polymyxins in gram-negative organisms. Int J Antimicrob Agents. 2017;49:526-35.

24. Caniaux I, van Belkum A, Zambardi G, Poirel L, Gros MFMCR. Modern colistin resistance. Eur J Clin Microbiol Infect Dis. 2017;36:415-20.

25. Mendes RE, Castanheira M, Farrell DJ, Flamm RK, Sader HS, Jones RN. Longitudinal (2001-14) analysis of enterococci and VRE causing invasive infections in European and US hospitals, including a contemporary (2010-13) analysis of oritavancin in vitro potency. J Antimicrob Chemother. 2016;71:3453-8.

26. Reyes K, Bardossy AC, Zervos M. Vancomycin-resistant enterococci: epidemiology, infection prevention, and control. Infect Dis Clin N Am. 2016;30:953-65.

27. Chiang H-Y, Perencevich EN, Nair R, et al. Incidence and outcomes associated with infections caused by vancomycin-resistant enterococci in the united states: systematic literature review and meta-analysis. Infect Control Hosp Epidemiol. 2017:38:203-15.

28. Bardet L, Le Page S, Rolain J-M. Milieu de culture sélectif polyvalent pour l'isolement et la sélection de bactéries résistantes à la colistine et à la vancomycine. 2016

29. Berrazeg M, Hadjadj L, Ayad A, Drissi M, Rolain J-M. First detected human case in algeria of $\mathrm{mcr}-1$ plasmid-mediated colistin resistance in a 2011 Escherichia coli isolate. Antimicrob Agents Chemother. 2016;60:6996-7.

30. Rolain J-M, Kempf M, Leangapichart T, et al. Plasmid-mediated mcr-1 gene in colistin-resistant clinical isolates of Klebsiella pneumoniae in France and Laos. Antimicrob Agents Chemother. 2016;60:6994-5.

31. Leangapichart T, Gautret $P$, Brouqui $P$, Mimish Z, Raoult D, Rolain J-M. Acquisition of mcr-1 plasmid-mediated colistin resistance in Escherichia coli and Klebsiella pneumoniae during hajj 2013 and 2014. Antimicrob Agents Chemother. 2016:60:6998-9.

32. Olaitan AO, Dia NM, Gautret $P$, et al. Acquisition of extended-spectrum cephalosporin- and colistin-resistant Salmonella enterica subsp. enterica serotype Newport by pilgrims during hajj. Int J Antimicrob Agents. 2015:45:600-4.

33. Diene SM, Merhej V, Henry M, et al. The rhizome of the multidrug-resistant Enterobacter aerogenes genome reveals how new "killer bugs" are created because of a sympatric lifestyle. Mol Biol Evol. 2013;30:369-83.

34. Olaitan AO, Rolain J-M. Interruption of $\mathrm{mgrB}$ in the mediation of colistin resistance in Klebsiella oxytoca. Int J Antimicrob Agents. 2015;46:354-5.

35. Bardet L, Baron S, Leangapichart T, Okdah L, Diene SM, Rolain J-M. Deciphering Heteroresistance to colistin in a Klebsiella pneumoniae isolate from Marseille, France. Antimicrob Agents Chemother. 2017;61:e00356-17.

36. Telke AA, Olaitan AO, Morand S, Rolain J-M. soxRS induces colistin heteroresistance in Enterobacter asburiae and Enterobacter cloacae by regulating the acrAB-to/C efflux pump. J Antimicrob Chemother 2017; published online July 25. DOI:10.1093/jac/dkx215.

37. Leangapichart T, Dia NM, Olaitan AO, Gautret P, Brouqui P, Rolain J-M. Acquisition of extended-spectrum b-lactamases by Escherichia coli and Klebsiella pneumoniae in gut microbiota of pilgrims during the hajj pilgrimage of 2013. Antimicrob Agents Chemother. 2016;60:3222-6.

38. Olaitan AO, Diene SM, Assous MV, Rolain J-M. Genomic plasticity of multidrug-resistant NDM-1 positive clinical isolate of Providencia rettgeri. Genome Biol Evol. 2015:8:723-8.

39. Rolain J-M, Diene SM, Kempf M, Gimenez G, Robert C, Raoult D. Real-time sequencing to decipher the molecular mechanism of resistance of a clinical pan-drug-resistant Acinetobacter baumannii isolate from Marseille, France. Antimicrob Agents Chemother. 2013;57:592-6.

40. Vila-Farrès $X$, Ferrer-Navarro $M$, Callarisa AE, et al. Loss of LPS is involved in the virulence and resistance to colistin of colistin-resistant Acinetobacter nosocomialis mutants selected in vitro. J Antimicrob Chemother. 2015:70. 2981-6.
41. Pailhoriès H, Hadjadj L, Mahieu R, Crochette N, Rolain J, Kempf M. Fortuitous diagnosis of NDM-1-producing Acinetobacter pittii carriage in a patient from France with no recent history of travel. J Antimicrob Chemother. 2017;72:942-4.

42. Launay A, Ballard SA, Johnson PDR, et al. Transfer of vancomycin resistance transposon Tn 1549 from Clostridium symbiosum to Enterococcus spp. in the gut of gnotobiotic mice. Antimicrob Agents Chemother. 2006;50:1054-62.

43. Rolain J-M, François P, Hernandez D, et al. Genomic analysis of an emerging multiresistant Staphylococcus aureus strain rapidly spreading in cystic fibrosis patients revealed the presence of an antibiotic inducible bacteriophage. Biol Direct. 2009;4:1

44. Chabou S, Leangapichart T, Okdah L, Le Page S, Hadjadj L. Rolain J-MM. Real-time quantitative PCR assay with Taqman* probe for rapid detection of MCR-1 plasmid-mediated colistin resistance. New Microbes New Infect. 2016;13:71-4.

45. Domingo M-C, Huletsky A, Giroux R, et al. High prevalence of glycopeptide resistance genes $v a n B$, vanD, and $v a n G$ not associated with enterococci in human fecal flora. Antimicrob Agents Chemother. 2005;49:4784-6.

46. Seng P, Drancourt M, Gouriet $F$, et al. Ongoing revolution in bacteriology : routine identification of bacteria by matrix-assisted laser desorption ionization time-of-flight mass spectrometry. Clin Infect Dis. 2009;49:543-51.

47. Postgate JR, Hunter JR. On the survival of frozen bacteria. J Gen Microbiol. 1961;26:367-78.

48. Hraiech $\mathrm{S}$, Roch A, Lepidi $\mathrm{H}$, et al. Impaired virulence and fitness of a colistin-resistant clinical isolate of Acinetobacter baumannii in a rat model of pneumonia. Antimicrob Agents Chemother. 2013;57:5120-1.

49. Choi M-J, Ko KS. Loss of hypermucoviscosity and increased fitness cost in colistin-resistant Klebsiella pneumoniae sequence type 23 strains. Antimicrob Agents Chemother. 2015;59:6763-73.

50. López-Rojas R, Domínguez-Herrera J, McConnell MJ, et al. Impaired virulence and in vivo fitness of colistin-resistant Acinetobacter baumannii. J Infect Dis. 2011;203:545-8.

51. Seng P, Rolain J-M, Fournier PE, La Scola B, Drancourt M, Raoult D. MALDITOF-mass spectrometry applications in clinical microbiology. Future Microbiol. 2010;5:1733-54.

52. Nordmann P, Jayol A, Poirel LA. Universal culture medium for screening polymyxin-resistant gram-negative isolates. J Clin Microbiol. 2016;54:1395-9.

53. Jayol A, Dubois V, Poirel L, Nordmann P. Rapid detection of polymyxinresistant Enterobacteriaceae from blood cultures. J Clin Microbiol. 2016;54: 2273-7.

54. Saly M, Jayol A, Poirel L, Megraud F, Nordmann P, Dubois V. Prevalence of faecal carriage of colistin-resistant gram-negative rods in a university hospital in western France, 2016. J Med Microbiol. 2017;66:842-3.

55. Abdul Momin MHF, Bean DC, Hendriksen RS, Haenni M, Phee LM, Wareham DW. CHROMagar COL-APSE: a selective bacterial culture medium for the isolation and differentiation of colistin-resistant Gram-negative pathogens. J Med Microbiol 2017; published online Oct 6. DOI:10.1099/jmm.0.000602.

56. Y-M A, Kim A-J, Lee J-Y. Colistin resistance in Klebsiella pneumoniae. Int J Antimicrob Agents. 2014:44:8-15.

\section{Submit your next manuscript to BioMed Central and we will help you at every step:}

- We accept pre-submission inquiries

- Our selector tool helps you to find the most relevant journal

- We provide round the clock customer support

- Convenient online submission

- Thorough peer review

- Inclusion in PubMed and all major indexing services

- Maximum visibility for your research

Submit your manuscript at www.biomedcentral.com/submit
) Biomed Central 